\title{
Implementation of SHA512 Hash Function and Boyer-Moore String Matching Algorithm for JPEG/exif Message Digest Compilation
}

\author{
Rachmad Fitriyanto ${ }^{1}$, Anton Yudhana ${ }^{2}$, Sunardi $^{3}$ \\ ${ }^{1}$ Magister of Informatics Engineering, Universitas Ahmad Dahlan, Yogyakarta, Indonesia \\ ${ }^{2,3}$ Electrical Engineering Department, Universitas Ahmad Dahlan, Yogyakarta, Indonesia \\ 11 fitriyanto7477@gmail.com, ${ }^{2}$ yudhana@ee.uad.ac.id, ${ }^{3}$ sunardi@mti.uad.ac.id
}

\begin{abstract}
Security information method for JPEG/exif documents generally aims to prevent security attacks by protecting documents with password and watermark. Both methods cannot be used to determine the condition of data integrity at the detection stage of the information security cycle. Message Digest is the essence of a file that has a function as a digital fingerprint to represent data integrity. This study aims to compile digital fingerprints to detect changes that occurred in JPEG / exif documents in information security. The research phase consists of five stages. The first stage, identification of the JPEG / exif document structure conducted using the Boyer-Moore string matching algorithm to find JPEG/exif segments location. The Second stage is segment content acquisition, conducted based on segment location and length obtained. The Third step, computing message digest for each segment using SHA512 hash function. Fourth stage, JPEG / exif document modification experiments to identified affected segments. The fifth stage is selecting and combining the hash value of the segment into the message digest. The obtained result shows the message digest for JPEG/exif documents composed of three hash values. The SOI segment hash value used to detect modifications for JPEG to png conversion and image editing. The APP1 hash value used to detect metadata editing. The SOF0 hash values use to detect modification for image recoloring, cropping and resizing - the combination from three hash values as JPEG/exif's message digest.
\end{abstract}

Keywords- Message digest, JPEG/exif, SHA512, Hash value, Boyer-Moore

\section{INTRODUCTION}

The JPEG/exif document is a document format that results from the use of digital cameras such as a smartphone camera. The JPEG/exif documents as image files are widely used in digital communications such as on social media. The Exchange of information requires security to ensure the information received is the same as the information sent. Information security for JPEG/exif documents generally designed to prevent document modifications. The password usages in the JPEG/exif document has long been used but can still be overcome with a variety of password remover tools that are widely available. The other technique for securing JPEG/exif is shown in the study by Wijayanto [1]. This study shows exif metadata data from JPEG/exif documents can be used to prevent copyright theft. The exif metadata provided information for digital analysis to detect altered JPEG/exif. Gangwar in [2] presented the methodology by comparing two exif metadata to analysis the data integrity of JPEG/exif's file. Another image file securing technique found in [3]. Int this research, the JPEG file secured by replacing selected parts of an image with another object. The exif metadata has secured before replacing the process beginning. The other technique beside the usages of exif metadata is the usage of watermark as an information security method in a study by Sukarno [4] to protect preventing document for modification. The purpose of watermark usage for JPEG/exif file security is to inform the attacker that the files protected by the physical presence of owner copyright [5]. The watermarks as a security method given robust protection for image geometry attack found in [6]. The research shows hidden and extracted watermark make the image files have more security the visible watermarks. The use of passwords, exif metadata, and watermark given great protection to prevent an attack on image files, but cannot be used in the detection stage of information security cycle to detect changes that occur in received JPEG/exif documents.

Message Digest is the essence of a file that can use to represent data integrity. The Message Digest is widely used to detect the integrity of data in installers provided by opensource application developers. The Message digest compiled using the hash function. The Hash function is a cryptographic method for the one-way encryption process. The output hash function is a hash value that has characteristics that cannot translate or decrypt into the original form [7]. The Hash value is very sensitive to changes in the input of any size. Secure Hash Algorithm (SHA) is a hash function developed by the National Institute of Science and Technology (NIST).

The characteristics from SHA that used for information security are one-way hash functions. This means that SHA generates a hash value that cannot decrypt [8]. Another SHA characteristic is this hash algorithm has high sensitivity for every input even a small change. Any modification or change in the input message will give different results [9]. 
SHA implementation for information security found in research by [8]. The SHA used to secure wireless communication by creating a message digest from the transmitted messages. The hash function in this research use the second generation of SHA. SHA consists of several categories that distinguished based on the size of the output hash value [10]. SHA variant from [8] use SHA-1 which has a 160-bit size of the hash value. The weakness from SHA-1 found in 2005 by Rijmen and Oswald [11]. The attack on SHA-1 made NIST developed the next generation of SHA. The variants of SHA produced SHA-2 with SHA256 and SHA512. Both variants in SHA-2 have longer hash value. Between two variants from SHA-2, SHA512 have better performance from SHA256. SHA512 has an output size of 512 bits that make this variant better than the previous hash function [11].

The use of SHA512 for information security was found in a study by Refialy [12]. This study uses SHA512 to compile the hash value of a pdf document. The obtained results indicate the hash value can detect small changes in the modification of pdf documents. The JPEG/exif file has a larger size than a pdf document. The size of the JPEG/exif document is the result of the development of optical technology in digital cameras. The larger the size of the document, the process of composing the message digest requires more time. This study aims to compile a concise message digest to detect changes in JPEG / exif documents in information security.

String matching algorithms are used to match or compare one or several characters and strings [13]. The Boyer-Moore string matching algorithm is included in the exact string matching algorithm that performs searches by comparing the characters of the strings tested with the pattern sought [14]. The implementation of the BoyerMoore string matching algorithm found in research by Ramos-Frías [15]. This research was presenting the usage of the Boyer-Moore string matching algorithm for enhanced data processing in the graphics processing unit (GPU). Another implementation is in medical science for human DNA searching [16][17].

The Boyer-Moore has two rules for the searching process, Good-Character rule, and Bad-Character Rule. These two rules determined the direction of the searching process. Bad-Character rule occurred if a character from the pattern is not

the same as in string. This condition will make a comparison shift to the left to next character of pattern. Good-Character rule occurred if a character from the pattern is the same as in string after Bad-Character rule occurred. This condition will make the next comparison shift right aligning two-match characters. Those two rules searching make the searching process faster than other exact string matching algorithms by avoiding notnecessary character comparison [18].

\section{METHOD}

A research study conducted in five stages that shown as in Figure 1. The first stage is the identification of the image file segment. This stage has the purpose to identify the JPEG/exif file structure. Image files as research object acquired from two smartphone types, Asus Z00UD, and Samsung Galaxy A5. Each smartphone takes 10 images. Images file taken with embedded camera apps from each smartphone with mode auto from indoor and outdoor sites.

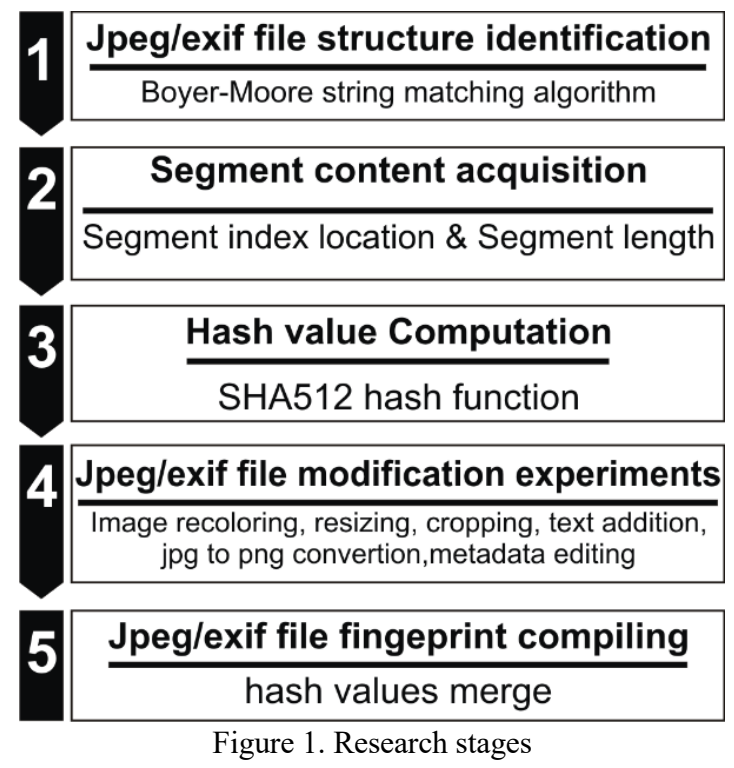

A result of the first stage is the location index of each file part. This location index will use on the second stage as a parameter to identify the beginning and end of file parts. Identification process conducted by use the BoyerMoore string matching algorithm for segment marker searching. Segment markers are data bit located at the beginning of each JPEG/exif segments [19]. JPEG/exif segment marker consists of four-bit hexadecimal. These bits have functioned as file structure signatures and contain specific information for computer and image viewer application to determine the suitable method to display image. Table 1 shows a segment marker value for each segment.

Table 1. JPEG/exif segment marker

\begin{tabular}{lc}
\hline \multicolumn{1}{c}{ Segment } & $\begin{array}{c}\text { Segment } \\
\text { Marker }\end{array}$ \\
\hline SOI (Start Of Image) & $\mathrm{ffd} 8$ \\
APP1 (Application-1) & $\mathrm{ffe1}$ \\
DQT (Define Quantization Table) & $\mathrm{ffdb}$ \\
SOF0 (Start Of Frame-0) & $\mathrm{ffc} 0$ \\
DHT (Define Huffman Table) & $\mathrm{ffc} 4$ \\
SOS (Start Of Scan) & $\mathrm{ffda}$ \\
\hline
\end{tabular}

Segmen structured as sequences as shown in Table 1 for the image file that has not altered. Each segment marker used as a pattern that sought in string matching algorithm. Boyer-Moore string matching algorithm start pattern searching from most-right pattern character and shift to left until reaching the leftmost character from pattern [20]. Figure 2 shown the Boyer-Moore flowchart.

Message Digest Compilation

(Rachmad Fitriyanto, Anton Yudhana, SUnardi) 


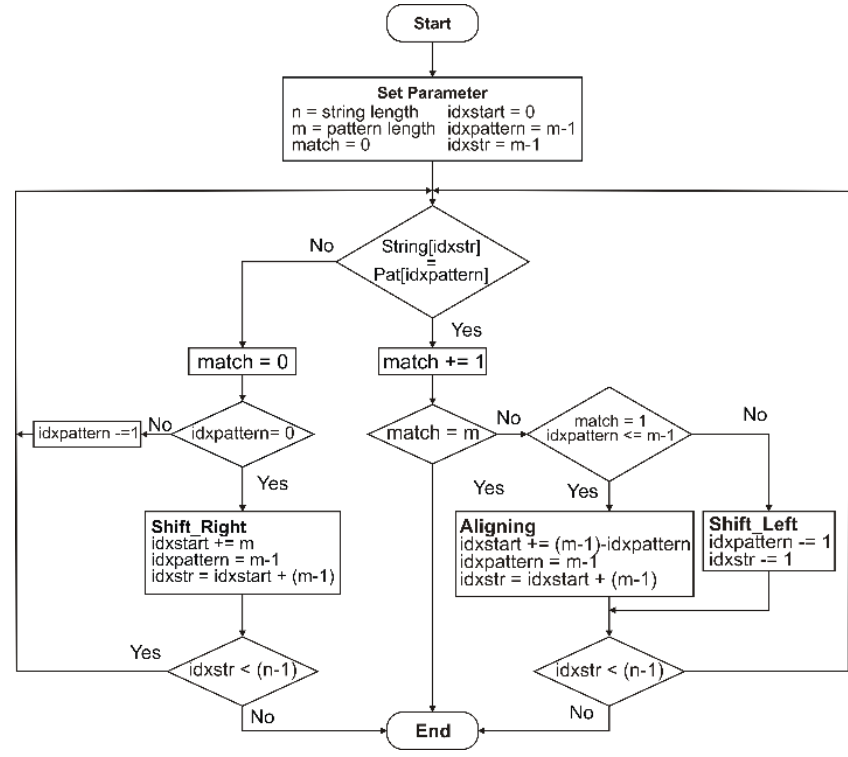

Figure 2. Boyer-Moore string matching flowchart

The Boyer-Moore string matching algorithm has two searching stages. The first stage is preprocessing, comprises of variable assignments such as $\mathrm{m}$ for pattern length, $\mathrm{n}$ for string length idxstr for string character index, idxpatter for pattern character index and match for character match comparison that occurred. The second stage is the character comparison. The second stage executed as an iteration loop that boundary by two conditions. Looping will stop if the match variable has valued the same as $m$ variable or comparison has reached the end of the string. The segment location index identified by the last value of idxstr variable.

The second stage is segment content acquisition. To acquire segment content, we need two parameters, starting index and length of content. The starting index provided by the segment index location. Segment length computes from subtraction between index location values from two adjacent segments. The third stage is the hash value computation. The computation conducted for every segment. Hash value computation with SHA512 hash function consists of three stages, preprocessing, hash computation and hash value compilation. Figure 3 show the sequences of the stages.

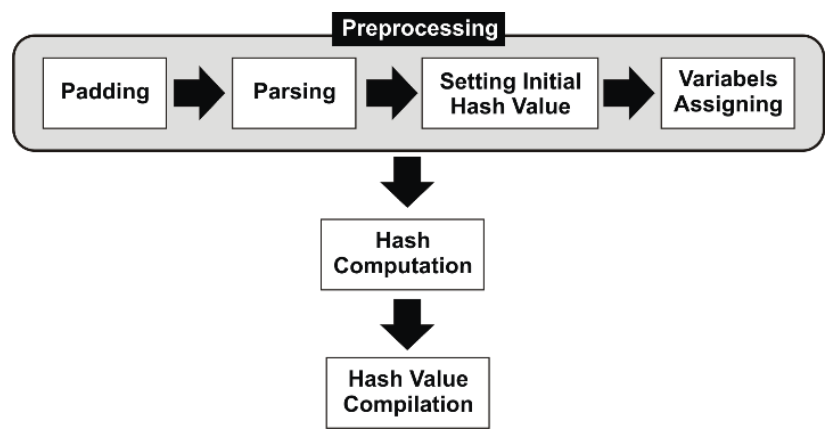

Figure 3. SHA512 computation stages

The preprocessing stages consist of four processes, padding, parsing, setting initial hash value and variable assigning [3]. The padding process is the adjustment of the size of input data so that the processed data has a size of multiples of 512 bits. The parsing process is to divide the data bits into groups of data with 64-bit size. The process of determining initial hash values and register assignments arranged as shown in Table 2.

Table 2. JPEG/exif segment marker

\begin{tabular}{clc}
\hline Register & & Hash Value \\
\hline $\mathrm{a}$ & $\mathrm{H}_{(0)}{ }^{0}$ & $=6 \mathrm{a} 09 \mathrm{e} 667 \mathrm{f} 3 \mathrm{bcc} 908$ \\
$\mathrm{~b}$ & $\mathrm{H}_{(1)}{ }^{0}$ & $=$ bb67ae8584caa73b \\
$\mathrm{c}$ & $\mathrm{H}_{(2)}{ }^{0}$ & $=3 \mathrm{c} 6 \mathrm{ef} 372 \mathrm{fe} 94 \mathrm{f} 82 \mathrm{~b}$ \\
$\mathrm{~d}$ & $\mathrm{H}_{(3)}{ }^{0}$ & $=\mathrm{a} 54 \mathrm{ff} 53 \mathrm{a} 5 \mathrm{f} 1 \mathrm{~d} 36 \mathrm{f} 1$ \\
$\mathrm{e}$ & $\mathrm{H}_{(4)}{ }^{0}$ & $=510 \mathrm{e} 527 \mathrm{fade} 682 \mathrm{~d} 1$ \\
$\mathrm{f}$ & $\mathrm{H}_{(5)}{ }^{0}$ & $=9 \mathrm{~b} 05688 \mathrm{c} 2 \mathrm{~b} 3 \mathrm{e} 6 \mathrm{c} 1 \mathrm{f}$ \\
$\mathrm{g}$ & $\mathrm{H}_{(6)}{ }^{0}$ & $=1 \mathrm{f} 83 \mathrm{~d} 9 \mathrm{abfb} 41 \mathrm{bd} 6 \mathrm{~b}$ \\
$\mathrm{~h}$ & $\mathrm{H}_{(7)}{ }^{0}$ & $=5 \mathrm{be} 0 \mathrm{~cd} 19137 \mathrm{e} 2179$ \\
\hline
\end{tabular}

The eight registers (a, b, c, d, e, f, g, h) and blocks of input data are used in computing the hash value according to the message expanding block sequence shown in Figure 4.

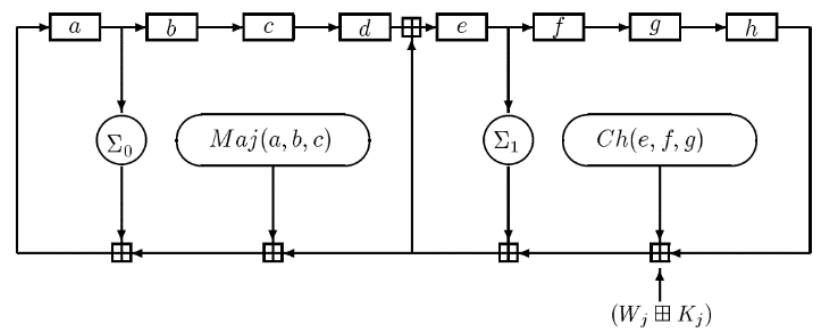

Figure 4. Message expanding block sequence diagram

The hash value computing executed iteratively as much as the block of data bits generated from the parsing stage. The mathematical equation in Figure 4 used according to what is shown in equations 1 to 6 .

$$
\begin{array}{rc}
\operatorname{Ch}(x, y, z) & =(x \wedge y) \oplus(\neg x \wedge z) \\
\operatorname{Maj}(x, y, z) & =(x \wedge y) \oplus(x \wedge z) \oplus(y \wedge z) \\
\sum 0(x) & =S^{28}(x) \oplus S^{34}(x) \oplus S^{39}(x) \\
\sum 1(x) & =S^{14}(x) \oplus S^{18}(x) \oplus S^{41}(x) \\
\sigma_{0}(x) & =S^{1}(x) \oplus S^{8}(x) \oplus R^{7}(x) \\
\sigma_{1}(x) & =S^{19}(x) \oplus S^{61}(x) \oplus R^{6}(x)
\end{array}
$$

The results of the computing process are eight hash values stored in eight registers. Figure 5 shows the results of calculating the hash value for the input "abc" string.

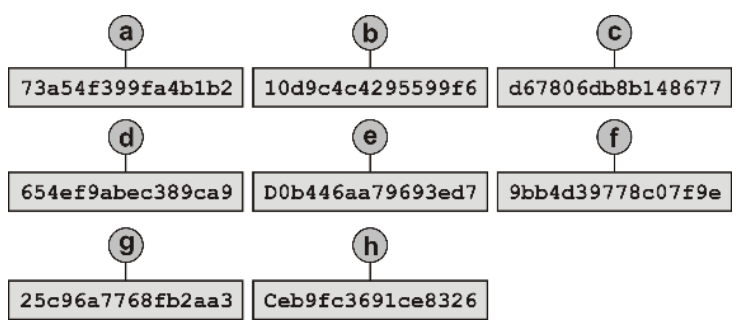

Figure 5. Hash value stored in eight registers

Each hash value in eight registers ( $a, b, c, d, e, f, g, h)$ then add with the initial hash value as shown in Figure 6. 
$\mathrm{H} 1=6 \mathrm{a} 09 \mathrm{e} 667 \mathrm{f} 3 \mathrm{bcc} 908+73 \mathrm{a} 54 \mathrm{f399fa} 4 \mathrm{~b} 1 \mathrm{~b} 2=$ ddaf35a193617aba $\mathrm{H} 2=\mathrm{bb} 67 \mathrm{ae} 8584 \mathrm{caa} 73 \mathrm{~b}+10 \mathrm{~d} 9 \mathrm{c} 4 \mathrm{c} 4295599 \mathrm{f} 6=\mathrm{cc} 417349 \mathrm{ae} 204131$ H3 = 3c6ef372fe94f82b +d67806db8b148677 = 12e6fa4e89a97ea2 $\mathrm{H} 4$ = a54ff53a5f1d36f1 + 654ef9abec389ca9 = 0a9eeee64b55d39a $\mathrm{H} 5=510 \mathrm{e} 527 \mathrm{fade} 682 \mathrm{~d} 1+\mathrm{d} 08446 \mathrm{aa} 79693 \mathrm{ed} 7=2192992 \mathrm{a} 274 \mathrm{fc} 1 \mathrm{a} 8$ $\mathrm{H} 6=9 \mathrm{~b} 05688 \mathrm{c} 2 \mathrm{~b} 3 \mathrm{e} 6 \mathrm{c} 1 \mathrm{f}+9 \mathrm{bb} 4 \mathrm{~d} 39778 \mathrm{c07f9e}=36 \mathrm{ba3c23a3feebbd}$ $\mathrm{H} 7=1 \mathrm{f} 83 \mathrm{~d} 9 \mathrm{abfb} 41 \mathrm{bd} 6 \mathrm{~b}+25 \mathrm{c} 96 \mathrm{a} 7768 \mathrm{fb} 2 \mathrm{aa} 3=454 \mathrm{~d} 4423643 \mathrm{ce} 80$ $\mathrm{H} 8=5 \mathrm{be0}$ cd19137e2179 + ceb9fc3691ce8326 $=2 \mathrm{a} 9 \mathrm{ac} 94 \mathrm{fa} 54 \mathrm{ca} 49 \mathrm{f}$

Figure 6. registers hash values and initial hash values

The result of the hash value of SHA512 is a combination of eight hash values from Figure 6 composed sequentially as shown in Figure 7.

ddaf35a193617aba cc417349ae204131 12e6fa4e89a97ea2 0a9eeee64b55d39a 2192992a274fcla8 36ba3c23a3feebbd 454 d4423643ce80e 2a9ac94fa54ca49f

Figure 7. SHA512 result

Research's third stage is modification experiments that consist of six types of file modification. This stage consists of three main steps as shown in Figure 8.

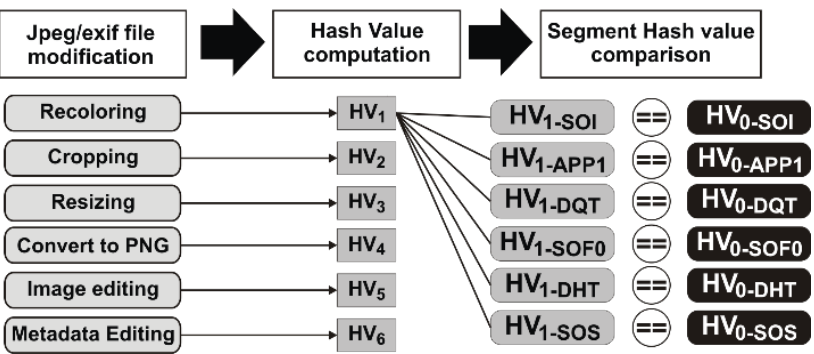

Figure 8. Image file modification experiments

Image recoloring, resizing, cropping conducted with ACDSee Pro.8 application. Metadata manipulation conducted with Hex Editor Neo. Image file format conversion from JPEG to png conducted by using FormatFactory application and image text addition conducted by using Paint application. This third stage has the purpose of identified altered segments caused by image modification. Identification conducted by comparing each segment's hash value from the original image with hash values from a modified image as shown in Figure 8. Before the image file modified, the hash value calculated from the original file $\left(\mathrm{HV}_{0}\right)$. The hash value of the original file used in comparison to the hash value of the modified file $\left(\mathrm{HV}_{1}\right.$, $\mathrm{HV}_{2}, \mathrm{HV}_{3}, \mathrm{HV}_{4}, \mathrm{HV}_{5}, \mathrm{HV}_{5}$, and HV6). Each modified form will have the value hash of each segment compiled.

The comparison of hash values conducted for each of the same segments. The hash value that has changed in each form of modification will be used as the compiler of the fingerprint file at a later stage. The last stage is a message digest compiling. Each hash value from the third stage compiles into one string file to form one message digest.

\section{RESULTS AND DISCUSSION}

Table 3 shows the image file size and picture mode, taken from smartphone Asus Z00UD.

\begin{tabular}{rcc}
\multicolumn{2}{c}{ Table 3. Image file from smartphone Asus Z00UD } \\
\hline JPEG/Exif file & $\begin{array}{c}\text { Size } \\
\text { (KB) }\end{array}$ & $\begin{array}{c}\text { Picture } \\
\text { Mode }\end{array}$ \\
\hline P_20180723_41211 & 2.117 & Auto/Outdoor \\
P_20180731_34049 & 1.915 & Auto/Outdoor \\
P_20180823_24724 & 2.488 & Auto/Indoor \\
P_20180905_85850 & 1.977 & Auto/Outdoor \\
P_20190110_00735 & 1.482 & Auto/Indoor \\
P_20190324_10003 & 1.601 & Auto/Indoor \\
P_20190324_10004 & 1.564 & Auto/Indoor \\
P_20190324_11402 & 2.315 & Auto/Indoor \\
P_20190324_11530 & 1.538 & Auto/Outdoor \\
P_20190324_12100 & 1.703 & Auto/Outdoor
\end{tabular}

The images data shown in Table 3, taken from two categories place, indoor and outdoor. These two locations did not affect the file size. The biggest file from Table 3 shows that images with multiple colors will make a file size bigger. Table 4 shows image files from the smartphone Samsung Galaxy A5.

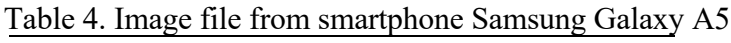

\begin{tabular}{ccc}
\hline JPEG/Exif file & $\begin{array}{c}\text { Size } \\
\text { (KB) }\end{array}$ & $\begin{array}{c}\text { Picture } \\
\text { Mode }\end{array}$ \\
\hline 01_20180825_131753 & 4.968 & Auto/Outdoor \\
02_20171225_111236 & 3.346 & Auto/Outdoor \\
03_20171201_124906 & 3.075 & Auto/Outdoor \\
04_20171201_130420 & 3.304 & Auto/Outdoor \\
05_20180825_134942 & 2.203 & AutoOutdoor \\
06_20181213_172235 & 1.208 & Auto/Indoor \\
07_20190114_154205 & 2.422 & Auto/Indoor \\
08_20190114_154209 & 2.101 & Auto/Indoor \\
09_20190114_154215 & 2.065 & Auto/indoor \\
10_20190114_154220 & 2.151 & Auto/indoor \\
\hline
\end{tabular}

The image files from the smartphone Samsung Galaxy A5 have different characteristics than image files from smartphone Asus Z00UD. The file size from Table 4 influenced by the amount of light and color captured in the image. The result of JPEG / exif file segment identification shown in Tables 3 and 4. Table 3 shown the segment location index for an image file from the Asus Z00UD smartphone.

Table 3. Segment index location for an image file from smartphone Asus Z00UD

\begin{tabular}{|c|c|c|c|c|c|c|}
\hline \multirow{2}{*}{$\begin{array}{c}\text { JPEG/Exif } \\
\text { file }\end{array}$} & \multicolumn{6}{|c|}{ Segment Index } \\
\hline & SOI & APP1 & DQT & SOF0 & DHT & SOS \\
\hline $\begin{array}{l}P_{-} 2018072 \\
3 \_41211\end{array}$ & 0 & 4 & 26400 & 26844 & 26726 & 27630 \\
\hline $\begin{array}{l}\text { P_2018073 } \\
\text { 1_34049 }\end{array}$ & 0 & 4 & 26368 & 61656 & 26694 & 27598 \\
\hline 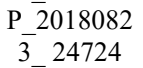 & 0 & 4 & 26378 & 26664 & 26704 & 27610 \\
\hline $\begin{array}{c}\mathrm{P}_{\overline{5}} \\
2018090 \\
85850\end{array}$ & 0 & 4 & 25350 & 25636 & 25676 & 26580 \\
\hline $\begin{array}{l}P_{-} 2019011 \\
0_{-} 00735\end{array}$ & 0 & 4 & 26318 & 26602 & 26642 & 27548 \\
\hline
\end{tabular}

Implementation SHA512 Hash Function And Boyer-Moore String Matching Algorithm For JPEG/exif

Message Digest Compilation

(Rachmad Fitriyanto, Anton Yudhana, SUnardi) 


\begin{tabular}{|c|c|c|c|c|c|c|}
\hline \multirow{2}{*}{$\begin{array}{c}\text { JPEG/Exif } \\
\text { file }\end{array}$} & \multicolumn{6}{|c|}{ Segment Index } \\
\hline & SOI & APP1 & DQT & SOF0 & DHT & SOS \\
\hline $\begin{array}{c}P_{-} 2019032 \\
10003\end{array}$ & 0 & 4 & 34212 & 25544 & 25584 & 26490 \\
\hline $\begin{array}{l}\mathrm{P}_{\overline{4}} \overline{2} \\
4 \\
10004\end{array}$ & 0 & 4 & 25378 & 25662 & 25704 & 26608 \\
\hline $\mathrm{P}_{\overline{4}} \mathrm{C}_{-} \mathbf{2} 019032$ & 0 & 4 & 25278 & 25769 & 25809 & 26713 \\
\hline 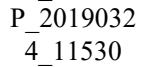 & 0 & 4 & 25348 & 25789 & 25792 & 26713 \\
\hline $\begin{array}{c}\mathrm{P}_{\overline{4}} \overline{2} \\
12190032\end{array}$ & 0 & 4 & 25405 & 25663 & 25564 & 26580 \\
\hline
\end{tabular}

by finding the difference in location index values from two adjacent segments as formulated in equation 7 .

$$
P_{\text {segmen }(n)}=\operatorname{Index}(n)-\operatorname{Index}(m)
$$

An example of the calculation of segment length for JPEG / exif documents from the Asus Z00UD smartphone shown in Table 5.

Segments index value obtained from variable idxstr that shown in Figure 2. Variable idxstr that uses is the last value before the looping process stopped. The location index of the SOI and APP1 segment in all JPEG / exif files in Table 3 have the same values 0 and 4 . This is because the SOI segment is located in the initial bit of the image file and consists of only four bits containing the segment marker segment, ffd8. The location index and length of the segments DQT, SOF0, DHT and SOS in ten JPEG / exif files have different values. This makes the index location of a segment of a JPEG / exif file not be used to identify the location of the segment in another file. Therefore, the Boyer-Moore algorithm matching string is always used to identify the location of the segment for each time the message digest compiled. The identification of the location of the JPEG / exif file segment from the Samsung Galaxy A5 smartphone shown in Table 4.

Table 4. Segment index location for an image file from smartphone Samsung Galaxy A5

\begin{tabular}{|c|c|c|c|c|c|c|}
\hline \multirow{2}{*}{$\begin{array}{l}\text { JPEG/Exif } \\
\text { file }\end{array}$} & \multicolumn{6}{|c|}{ Segment Index } \\
\hline & SOI & APP1 & DQT & SOF0 & DHT & SOS \\
\hline $\begin{array}{l}01 \_201808 \\
25 \_131753\end{array}$ & 0 & 4 & 2000 & 2286 & 2326 & 3218 \\
\hline $\begin{array}{l}02 \_201712 \\
25 \_111236\end{array}$ & 0 & 4 & 2000 & 2286 & 2326 & 3218 \\
\hline $\begin{array}{l}03 \_201712 \\
01 \_124906\end{array}$ & 0 & 4 & 2000 & 2286 & 2326 & 3218 \\
\hline $\begin{array}{l}04 \_201712 \\
01 \_130420\end{array}$ & 0 & 4 & 2000 & 2286 & 2326 & 3218 \\
\hline $\begin{array}{l}\text { 05_201808 } \\
25 \_134942\end{array}$ & 0 & 4 & 2000 & 2286 & 2326 & 3218 \\
\hline $\begin{array}{l}06 \_201812 \\
13 \_172235\end{array}$ & 0 & 4 & 2000 & 2286 & 2326 & 3218 \\
\hline $\begin{array}{l}07 \_201901 \\
14 \_154205\end{array}$ & 0 & 4 & 2000 & 2286 & 2326 & 3218 \\
\hline $\begin{array}{l}08 \_201901 \\
14 \_154209\end{array}$ & 0 & 4 & 2000 & 2286 & 2326 & 3218 \\
\hline $\begin{array}{l}09 \_201901 \\
14 \_154215\end{array}$ & 0 & 4 & 2000 & 2286 & 2326 & 3218 \\
\hline $\begin{array}{l}10 \_201901 \\
14 \quad 154220\end{array}$ & 0 & 4 & 2000 & 2286 & 2326 & 3218 \\
\hline
\end{tabular}

The location index of the SOI and APP1 segment in all JPEG / exif files in Table 4 has the same values 0 and 4 . This is because the SOI segment is located in the initial bit of the image file and the segment location index on the JPEG/exif file from the Samsung Galaxy A5 smartphone shows that each file has the same segment location. Therefore, identification of the location of the JPEG/exif segment that will be conducted in the future does not require a search from the start.

The location index of Tables 3 and 4 used to calculate the length of each segment. This calculation accomplished

Table 5. Image file Segment length

\begin{tabular}{|c|c|c|c|c|c|c|}
\hline \multirow{2}{*}{ File name } & \multicolumn{6}{|c|}{ Segment Length (bit) } \\
\hline & SOI & APP1 & DQT & SOF0 & DHT & SOS \\
\hline $\begin{array}{c}\text { P_20180723 } \\
\_141211\end{array}$ & 4 & 26396 & 444 & 118 & 904 & 9181254 \\
\hline $\begin{array}{l}\text { P_20180823 } \\
-124724\end{array}$ & 4 & 26374 & 286 & 40 & 906 & 5386020 \\
\hline $\begin{array}{l}\text { P_20180905 } \\
\text { _085850 }\end{array}$ & 4 & 25346 & 286 & 40 & 904 & 5319634 \\
\hline $\begin{array}{c}\text { P_20190324 } \\
\quad 100013\end{array}$ & 4 & 34208 & 8668 & 40 & 906 & 3197654 \\
\hline $\begin{array}{c}\text { P_20190324 } \\
\text { _100202 }\end{array}$ & 4 & 63584 & 38180 & 40 & 906 & 5009834 \\
\hline $\begin{array}{c}\text { 01_2018082 } \\
\text { 5_131753 }\end{array}$ & 4 & 1996 & 286 & 40 & 892 & 10805918 \\
\hline $\begin{array}{c}\text { 02_2017122 } \\
5 \_111236\end{array}$ & 4 & 1996 & 286 & 40 & 892 & 7275684 \\
\hline $\begin{array}{c}\text { 03_2017120 } \\
\text { 1_124906 }\end{array}$ & 4 & 1996 & 286 & 40 & 892 & 6686880 \\
\hline $\begin{array}{c}04 \_2017120 \\
1 \_130420\end{array}$ & 4 & 1996 & 286 & 40 & 892 & 6598378 \\
\hline $\begin{array}{c}05 \_2018082 \\
5-134942\end{array}$ & 4 & 1996 & 286 & 40 & 892 & 4788372 \\
\hline
\end{tabular}

The APP1 segment length in the first file in Table 3 has a value of 26396 obtained using equation 7 , which is the result of a reduction between the DQT segment location index and the APP segment1. The results of the long calculation of all segments show that the SOS segment has the largest segment length. This is because the SOS segment contains image data that is the main data from the Image file. The bigger the size of the JPEG / exif document, the longer the SOS segment will be. The calculation of SOS segment length accomplished by operating a subtraction between the overall image bit length and the SOS segment location index. The bit length of image data, obtained from the length calculation of the converted file at the beginning of the identification phase of the segment location that is also stored in the $n$ variable in the searching process with the Boyer-Moore string matching algorithm.

Figure 9 shows the application interface for content acquisition and calculation of hash values for each segment. Segment content acquired use location index value and segment length as boundary parameters. Location index to identify an acquisition starting point and segment length for numbers of characters that should acquire 


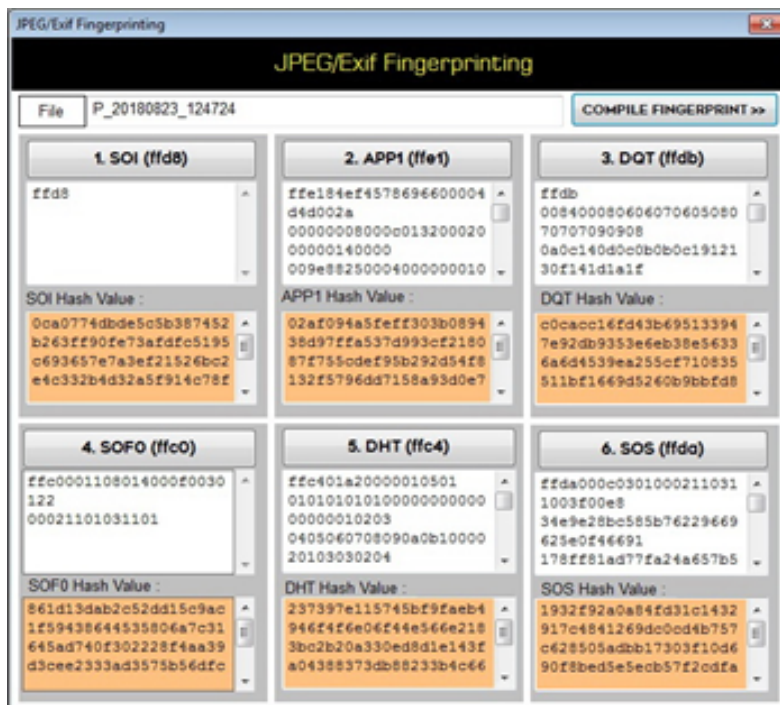

Figure 9. segment content acquisition and hashing

Six hash values from six JPEG/segments form figure 9 can use as message digest elements that will conduct on research fourth stage.

The research's fourth stage begins with modifying the image file. The modification process consists of six type experiments. The first modification is recoloring image, change the image file into a grayscale image as shown in Figure 10.

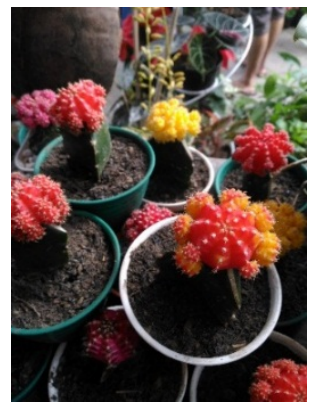

(a)

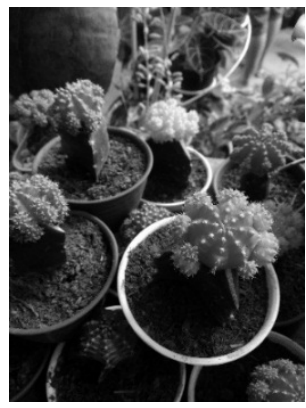

(b)
Figure 10. Example of recoloring image (a).Original image (b).Modified image

Recoloring image conducted shown from Figure 10, uses ACDSee Pro. 8 application. The Second modification is metadata editing. This experiment conducted using the Neo Hex editor application to change smartphone type inside the APP1 segment. Figure 11 shows a metadata comparison between the original and modified image.

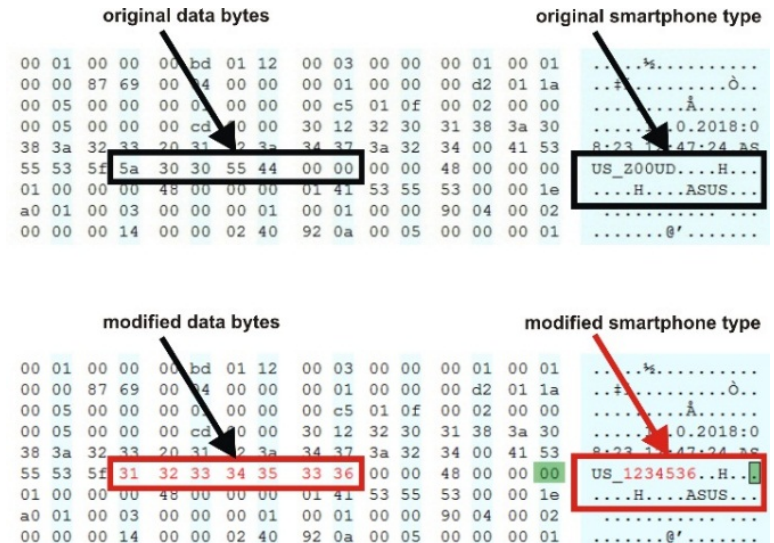

Figure 11. Example of metadata editing

Metadata editing from Figure 11, modified smartphone type from "Z00UD" into "123456". The modification conducted by change the original data bytes 5a 30305544 00 into 313233343536 in hexadecimal number format. The result from this modification is the metadata section has altered while the image still can be displayed.

The third experiment selects a part of an image using the ACDSee Pro.8 application. Figure 12 shows a comparison between the original image and the cropped image.

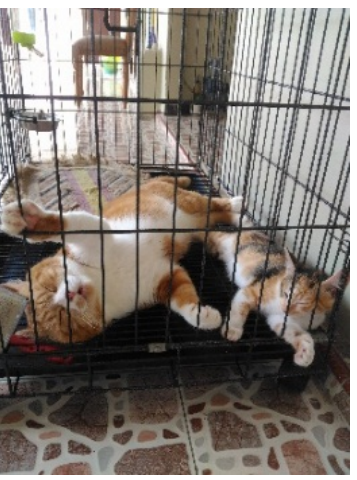

(a)

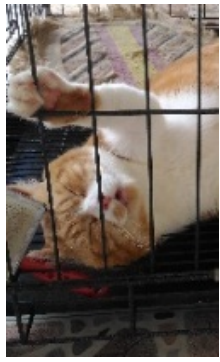

(b)
Figure 12. Example of cropping image (a).Original image (b).Cropped image

The fourth modification experiment is resizing. Figure 13 shows a comparison between the original and modified image.

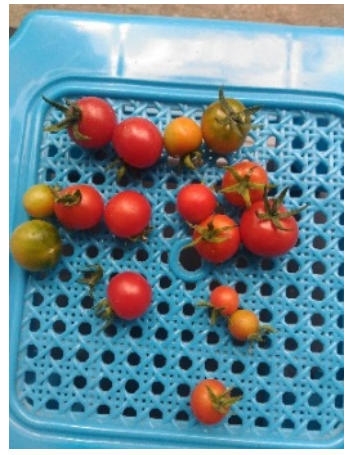

(a)

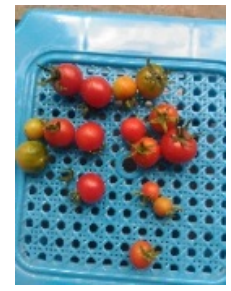

(b)

Figure 13. Example of resizing image

Message Digest Compilation

(Rachmad Fitriyanto, Anton Yudhana, SUnardi) 
(a).Original image (b).Modified image

Image dimension (width-height) on the fourth modification experiment, reduced into $50 \%$ smaller from the original image. This modification conducted uses ACDSee Pro. 8 application.

The fifth modification is file type conversion. The original file type is JPEG/exif, converted into png uses Format Factory application. The conversion result did not show the significance result from the displayed image. The differences clearly are shown if the converted image opened in the hex editor application as shown in Figure 14

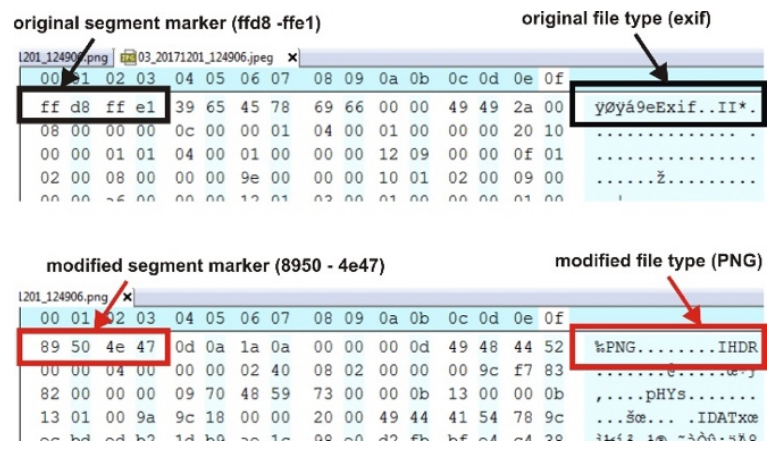

Figure 14. Example of a comparison image's data bytes from the converted file

The file type from the two files the different values. The original file has exif type while the modified file has png file type. The change of file type made identified by searching the file signature from both files. The file signature used by the computer to determine how to open a file. The file signature located at the beginning of the data file. The original segment marker on JPEG/exif file type, have file signatures that identified as ffd8. File type conversion on this modification experiments, not just affected the change of file signature only, but also altered the entire data bytes of the image file. This condition caused by the changed of the file structure.

The sixth image modification is text addition. This experiment conducted by uses Core Photo-Paint X3 . Figure 15 shows a comparison between the original image and the modified image. The experiment conducted by add text into the image.

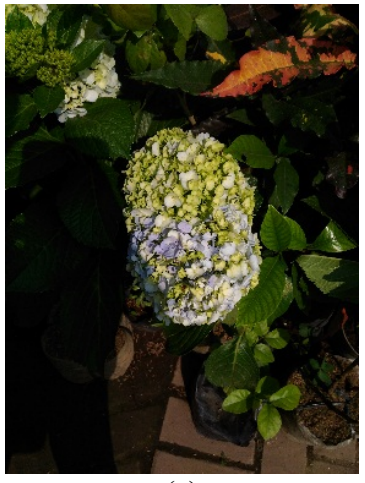

(a)

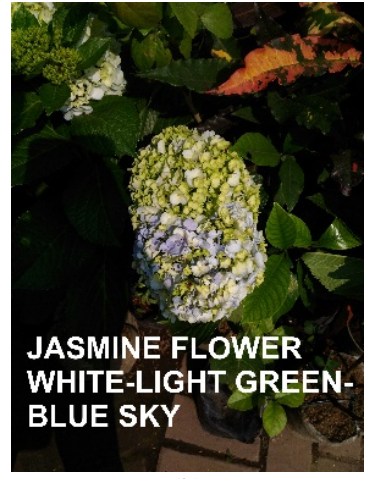

(b)
Figure 15. Example of text modification on the image file (a).Original image (b).modified image
Each original and modified image processed with SHA512 to produce hash values from each segment inside each image file. The obtained hash value then compared between the original and modified images. Figure 16 shows hash values comparison from recoloring image modification.

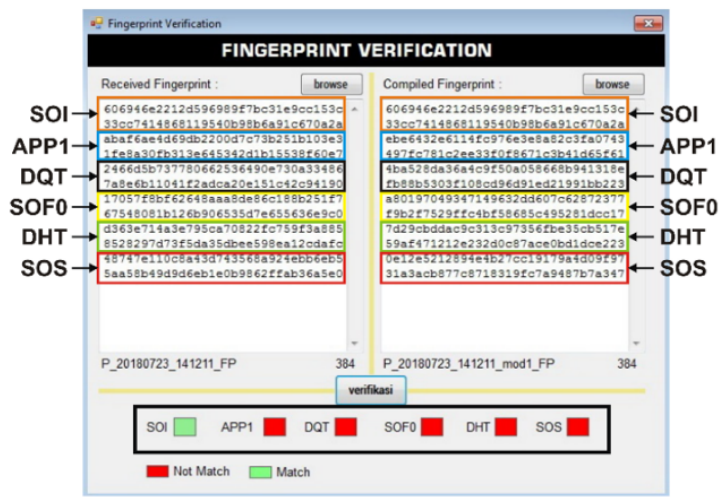

Figure 16. Application interface for hash values comparison

The left section of Figure 16 shows the hash value of six segments from the original file. The right section shows the hash value of five segments from the recoloring modification file. The comparison conducted by comparing two hash values from the same segment. The comparison results consist of two conditions, "Match" if the hash values of the two segments are equal and "Not match" if not the same. The comparison result from Figure 16 shows, only the value of the SOI segment hash value that altered. These results show that four other segments' content altered when recoloring modifications occurred. The affected segments from each modified experiments shown in Table 6 . The result from Table 6 categorized into three groups. The first group has shown that the metadata modification experiment affected the APP1 segment only. The second group showed that the SOI segment altered for image file conversion and text/object addition experiments.

Table 6. Affected segment for image modification

\begin{tabular}{ccccccc}
\hline Modification & \multicolumn{6}{c}{ Affected Segment } \\
\cline { 2 - 6 } Experiments & SOI & APP1 & DQT & SOF0 & DHT & SOS \\
\hline Recoloring & - & $\checkmark$ & $\checkmark$ & $\checkmark$ & $\checkmark$ & $\checkmark$ \\
Metadata Modification & - & $\checkmark$ & - & - & - & - \\
Resizing & - & $\checkmark$ & $\checkmark$ & $\checkmark$ & $\checkmark$ & $\checkmark$ \\
Convert to PNG & $\checkmark$ & $\checkmark$ & $\checkmark$ & $\checkmark$ & $\checkmark$ & $\checkmark$ \\
Text addition & $\checkmark$ & $\checkmark$ & $\checkmark$ & $\checkmark$ & $\checkmark$ & $\checkmark$ \\
Cropping & - & $\checkmark$ & $\checkmark$ & $\checkmark$ & $\checkmark$ & $\checkmark$ \\
\hline
\end{tabular}

The third group shown all segments altered for image display modification (recoloring, resizing, cropping). Each result group used as a base to determine the file fingerprint components as JPEG/exif message digest. The first component is SOI hash value from the first group and the second component is APP1 hash value from the second group. Third component selected from four segments (DQT, SOF0, DHT, SOS) based on segment content and segment length.

SOS segment has the most length content from other segments because this segment stored the image data. This condition made the segment marker searching process need more time because the Boyer-Moore string matching 
algorithm has $\mathrm{O}(\mathrm{mn})$ time complexity for worst-case conditions. SOF0 has the smallest size than others except for the SOI segment. SOF0 store image information such as image dimension and the number of color components that always change if image altered as in 1st, 2nd, 5th and 6th experiments. Figure 17 shown JPEG/exif file message digest that arranged from three hash values, SOI, APP1, and SOF0.

\begin{tabular}{|c|c|}
\hline ish Valt & $\begin{array}{l}\text { Oca0774dbde } 5 \text { c5b387452b } 263 f f 90 f e 73 a f d f c 51 \\
95 c 693657 \text { e } 7 \text { a ef2 } 21526 b c 2 e 4 c 332 b 4 d 32 a 5 f 91 \\
4 c 78 f 941018 a 4 d a 4 d 03 a e 4 d 993 c f 637 c 55 e 58 b 8\end{array}$ \\
\hline & \\
\hline $\begin{array}{l}\text { SOP } \\
\text { sh Valt }\end{array}$ & $\begin{array}{l}\text { 12f731ddc1749bc91e102e65988c01c2b6361b } \\
4 a 4477 b 51 \text { dacd45705eb8cbc0cacc16fd43b69 } \\
5133947 \text { e92db9353e6eb38e56336a6d4539ea } \\
255 c f 710835511 \text { bf } 1669 \text { d5260b9bbfd8a830725 } \\
7 \text { ee } 901549218466 \text { b269ca5755a9233ec0e342 }\end{array}$ \\
\hline
\end{tabular}

Figure 17 JPEG/exif message digest

Hash value from each segment in Figure 17, has 128bit length. This caused by number format from an application is in hexadecimal which in binary can convert to 512 -bit length by computing $128 \times 4$.

\section{CONCLUSION}

JPEG/exif message-digest consist of three hash values that represent modified experiments. SOI hash values use for identifying file conversion and text addition modification. APP1 hash values use for identifying metadata editing modification and SOF0 hash value for identifying recoloring, resizing and cropping modification. Identifying that three-segment will make segments searching faster for the Boyer-Moore string matching algorithm. The compilation of Message digest from SHA512 hash value has an advantage because of its small size and cannot decrypt. Future research hope can develop message digest compilation for other file types that common use in digital communication such as video and audio.

\section{REFERENCES}

[1] H. Wijayanto, I. Riadi, and Y. Prayudi, "Encryption EXIF Metadata for Protection Photographic Image of Copyright Piracy," Int. J. Res. Comput. Commun. Technol., vol. 5, no. 5, 2016.

[2] D. P. Gangwar and A. Pathania, "Authentication of Digital Image using Exif Metadata and Decoding Properties," Int. J. Sci. Res. Comput. Sci. Eng. Inf. Technol., vol. 3, no. January, pp. 335-341, 2019.

[3] L. Yuan and T. Ebrahimi, "Image Privacy Protection with Secure JPEG ransmorphing," Inst. Eng. Technol. Journals, vol. 11, no. 9, pp. 1-8, 2017.

[4] A.S.Sukarno, "Pengembangan Aplikasi Pengamanan Dokumen Digital Memanfaatkan Algoritma Advance Encryption Standard, RSA Digital Signature dan Invisible Watermarking," Pros. Semin. Nas. Apl. Teknol. Inf. 2013, pp. 1-8, NaN-5022, 2013.

[5] A. Chauhan, "Digital Watermarking-Revisit," Int. J. Comput. Sci. Inf. Technol., vol. 6, no. 1, pp. 833-838, 2015.

[6] D. Vaishnavi and T. S. Subashini, "A Secure and Robust Image Watermarking System Using Normalization and
Arnold Scrambling,” Int. J. Netw. Secur., vol. 18, no. 5, pp. 832-841, 2017.

[7] C. B. Shah and D. R. Panchal, "Secured Hash Algorithm-1: Review Paper," Int. J. Adv. Res. Eng. Technol., vol. 2, no. 10, pp. 26-30, 2014.

[8] N. Tiwari and A. Sinhal, "An Implementation on Secure Hash Algorithm in Wireless Algorithms to Ensure the Integrity," Int. J. Comput. Sci. Inf. Technol., vol. 5, no. January, pp. 4779-4781, 2014.

[9] N. C. Iyer and S. Mandal, "Implementation of Secure Hash Algorithm-1 using FPGA,” Int. J. Inf. Comput. Technol., vol. 3, no. 8, pp. 757-764, 2013.

[10] NIST, FIPS PUB 180-4 Secure Hash Standard ( SHS ), no. August. Gaithersburg: National Institute of Standards and Technology, 2015.

[11] I. Riadi and M. Sumagita, "Analysis of Secure Hash Algorithm (SHA) 512 for Encryption Process on Web Based Application," Int. J. Cyber-Security Digit. Forensics, vol. 7, no. 4, 2018.

[12] L.Refialy, E.Sediyono, and A.Setiawan, "Pengamanan Sertifikat Tanah Digital Menggunakan Digital Signature SHA-512," JUTISI, vol. 1, pp. 229-234, 2015.

[13] N. Jiji and T. Mahalakshmi, "Survey of Exact String Matching Algorithm for Detecting Patterns in Protein Sequence," Adv. Comput. Sci. Technol., vol. 10, no. 8, pp. 2707-2720, 2017.

[14] K. Al-Khamaiseh and S. Al-Shagarin, "A Survey of String Matching Algorithms," Int. J. Eng. Res. Appl., vol. 4, no. June 2015, pp. 144-156, 2014.

[15] R. Ramos-frí and M. Vargas-lombardo, "A Review of String Matching Algorithms and Recent Implementations using GPU," Int. J. Secur. Its Appl., vol. 11, no. 6, pp. 6976, 2017.

[16] J. Bhandari and A. Kumar, "String Matching Rules Used By Variants of Boyer-Moore Algorithm," J. Glob. Res. Comput. Sci., vol. 5, no. 1, pp. 8-11, 2014.

[17] Y. D. Prabowo, "Pencocokan DNA NR_108049 dan DNA DI203322 Menggunakan Algoritma Boyer Moore," Pros. Semin. Nas. Teknol. Inf. dan Komun., no. 40, pp. 18-19, 2016.

[18] D. R. Candra and K. D. Tania, "Application of Knowledge Sharing Features Using the algorithm Boyer-moore On Knowledge Management System (KMS)," J. Sist. Inf., vol. 9, no. 1, pp. 1216-1221, 2017.

[19] A. L. . Sandoval, D. M. . Gonzales, L. J. . Villaba, and J. Hernandez-Castro, "Analysis of errors in exif metadata on mobile devices," Multimed Tools Appl, no. 74, pp. 47354763, 2015.

[20] N. Jiji and T. Mahalaksmi, "An Efficient String Matching Algorithm for Detecting Pattern Using Forward and Backward Searching Approach," Int. J. Comput. Sci., vol. 6, no. 2, pp. 16-26, 2018.

Message Digest Compilation

(Rachmad Fitriyanto, Anton Yudhana, SUnardi) 